\title{
DTN Support for News Dissemination in an Urban Area
}

\author{
Tuan-Minh Pham and Serge Fdida \\ Laboratoire d'Informatique de Paris 6 (LIP6), \\ UPMC Sorbonne Universités, \\ 4 place Jussieu, 75005 Paris, France \\ \{tuan-minh.pham, serge.fdida\}@lip6.fr
}

\begin{abstract}
We are studying the practicality of news dissemination over a Delay Tolerant Network (DTN) in an urban area. The target application is the distribution of the electronic version of a newspaper in a large city. Therefore, although strict time constraints do not apply, spreading the information should be achieved within a reasonable delay. We consider that mobile users subscribe to their content of interest and expect to receive it within their journey from their home to their office. We provide two contributions. Firstly, we consider a simple DTN environment when content is distributed solely through inter-contact of mobile nodes. We derive analytical expressions for the packet delay in such environments and suggest how to improve effectively the expected message delay in the case of an area with low or high density of mobile nodes. Secondly, if the delay is found to be excessive, we suggest the deployment of some data kiosks in the environment to better support the dissemination of content. Data kiosks are simple devices that receive content directly from the source, usually using wired or cellular networks. We investigate both an upper bound and a lower bound of the number of data kiosks to distribute the content over a geographical area within an expected delay objective. We also show the important property that those bounds scale linearly with the contact rates between a mobile node and a data kiosk. The analytical results are validated through simulation using a number of mobility models.
\end{abstract}

Keywords: Hybrid DTN, delay, news dissemination, modeling, performance analysis.

\section{Introduction}

Free daily newspapers have been introduced in many countries worldwide for over 10 years now. Their distribution channel uses point of presence (Kiosk) often located at the entrance to the main transportation systems, such as metro or suburban train stations. They also have a presence on the web and exploit user's contributions and social networks. In addition, the widespread deployment of handheld devices provides the opportunity to use them for content distribution instead of being charged for cellular access. In the context of our research, we

J. Domingo-Pascual et al. (Eds.): NETWORKING 2011, Part I, LNCS 6640, pp. 120-133, 2011.

(C) IFIP International Federation for Information Processing 2011 
consider that mobile users will agree to contribute to such an application only for content of their own interest. Information conveyed by free daily newspapers does not need to be instantaneous. However, owners of these newspapers expect that the information will reach the reader within a time window related to the period he/she will spend commuting from their home to their place of work as this is the best time to capture their attention. The contribution of our paper is not restricted to the distribution of news as many other applications will exhibit similar expectations, but it provides a practical use case.

In this paper, we investigate the practicality of news dissemination over a DTN in an urban area. In DTNs, nodes can move freely and exchange content when they are within each other's transmission range. Since an end-to-end path is not available most of the time, a store-carry-forward paradigm is usually used to enable communication. Several questions arose in the above setting in order to design a system that achieved the required service while avoiding deploying too many digital data kiosks. A first step would be to develop a quantitative analysis to investigate the message delay of DTNs. What are the most sensitive parameters: readers' interest in content or contact opportunities? How effective can a DTN be without infrastructure? If the delay is found to be excessive, we suggest deploying some data kiosks in the environment to better support the dissemination of content. Data kiosks are simple devices that receive the content directly from the source, generally using wired or cellular networks. The question is how many data kiosks one has to invest in to satisfy performance constraints and where these data kiosks should be located. We are mostly interested in two performance metrics, the spreading time and the message delay. The message delay is the time needed to transmit content from a mobile node to another node, while the spreading time is the delay required to deliver the content to the last node in a group of nodes, or the time needed for the content to spread over a part of the network. The main question addressed in this paper is to determine the number of data kiosks necessary to disseminate content to a set of mobile nodes, when taking into account the spreading time in a given area and mobility pattern.

Our contribution is to provide explicit solutions to answer the above questions. To the best of our knowledge, our work is the first to obtain explicit expressions for the evaluation of the number of data kiosks needed to reduce the expected spreading time to a target requirement in opportunistic networks. We first investigate both a closed-form formulae and an asymptotic expression of the expected message delay in DTNs where mobile users share their contents only if they share the same interests. We show that the expected message delay is in the order of several hours even if some parameters are optimistic. Second, we consider a hybrid environment and derive closed-form expressions for both an upper and a lower bound of the number of data kiosks needed to satisfy the requirement of the expected spreading time. The results show how the contact rate between a mobile node and a data kiosk influences the quality of service parameters. 
The remainder of this paper is structured as follows. In the following section, we describe some related work. In Section 3, we analyze the system formally and derive the main results. In Section 4 , we present the analytical model to compute the number of data kiosks. In Section 5, we validate our solution against simulation under three mobility models and give a specific example when applying our results to improve the delay. Finally, Section 6 concludes the paper.

\section{Related Work}

DTN application to content dissemination has been contemplated with different perspectives. Lenders et al. developed a mobile distribution system where podcasting protocols are extended to the ad hoc domain [11. Garyfalos and Almeroth designed a system in advertising with a incentive scheme for data sharing through opportunistic contact [5]. In addition, McNamara et al. concentrate on the application of DTN in the context of the movement of people in transit systems due to the fact that people consume time on public transport [13].

The emergence of DTNs has triggered a considerable amount of work to help us to better understand their behavior and improve their performance. Contact opportunities have a strong impact on the performance of DTN and therefore, several contributions have focused on characterizing the inter-contact time distribution. Observing some real world traces, Karagiannis et al. investigated exponential tail behavior of inter-contact times [9]. Studying common mobility models, Groenevelt et al. discovered that the inter-contact time between mobile nodes is almost exponentially distributed [6]. Ibrahim et al., in addition, showed that the inter-contact time between mobiles and stationary nodes is also almost exponential in common mobility models such as the random waypoint or random direction [7.

Based on existing knowledge about contact opportunities, different papers analyzed the performance of DTNs with various assumptions on content distribution schemes including the unrestricted multicopy protocols [6], spray-and-wait [19, k-hop relay [18. Starting from 6], Hanbali et al. 3] extended the work with lifetime constraints and Zhang et al. 21] studied variations of the epidemic protocol and recovery schemes. Different measures were considered: throughput [2], delay 6321, content age [8].

Recent work was motivated by improving the performance of DTNs. Polat et al. proposed an algorithm to find mobile nodes that can act as message ferries [16. Another approach is to add some infrastructure nodes. This solution has been used in the context of ad hoc networks where nodes are fixed [122017]. In the DTN's framework, some papers estimated the average message delay in the network with the presence of infrastructure for multi-copy two hop routing protocol and epidemic routing protocol [7, .

Our work is different as it considers the probable effect of interest for a given content in estimating the expected message delay in DTNs. In addition, it computes the number of infrastructure nodes to meet a performance objective for expected spreading time. 


\section{System Description and Main Results}

We consider a system composed of $n$ mobile nodes and $k$ data kiosks. Mobile nodes are wireless devices carried by people, and data kiosks are stationary nodes in which a content update is downloaded via an infrastructure network (wired or cellular), to be stored and disseminated. We define mobile nodes that subscribe to a given content as subscribers. Subscribers are organized into healthy nodes and infected nodes. An infected (resp. healthy) node is a subscriber that has (resp. does not have) a copy of the content he subscribed to. We assume that every node has the same transmission range, interference from other nodes is negligible, and transmission is always successful and instantaneous in a sparse network.

The dissemination of news relies on opportunistic contacts and a store-carryand-forward paradigm. Motivated by 617, the pairwise inter-contact times for mobile-mobile (resp. mobile-data kiosk) contacts can be approximately represented as exponentially distributed random variables with mean $1 / \lambda, \lambda>0$ (resp. $1 / \mu, \mu>0$ ). All these random variables are assumed to be homogeneous and mutually independent. A healthy node will be infected when it meets either a data kiosk or an infected node, and it is interested in the news. We assume that a healthy node can change its interest at any time by subscribing to the content with a probability $\delta(0<\delta \leq 1)$ or unsubscribing with a probability $1-\delta$, and that only healthy nodes can change their interest when they meet other mobile nodes. One can expect that infected nodes will share their content during their journey as they will mostly read it during that time. We refer to this scheme as a user-preferred content distribution scheme.

\subsection{Expected Message Delay}

Since a service provider wishes to know whether or not adding some data kiosks is necessary, our first main result addresses the question of performance in a homogeneous environment when no data kiosk exists. The message delay $D$ is our preferred metric and is defined as the time required to deliver information (news/content) from a source to a destination node. It is expected that the destination node will keep its interest until it receives the content. We investigate both a closed-form expression and an asymptotic representation of the expected message delay as a function of $o(\delta)$ when $\delta$ is small. We are interested in the influence on the expected message delay of parameters such as the interest of people and contact rates.

Proposition 1. Under the user-preferred content distribution scheme, the expected message delay is given by

$$
\begin{aligned}
E[D] & =\frac{1}{\lambda} \sum_{i=1}^{n-1}\left(P\left\{N_{2}=i\right\} \sum_{j=1}^{i} \frac{1-(1-\delta)^{n-j}}{\delta j(n-j)}\right) \\
& =\frac{1}{\lambda}\left(1-\frac{n-2}{4} \delta\right)+o(\delta), \quad \text { for } \delta \rightarrow 0
\end{aligned}
$$




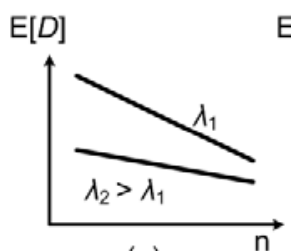

(a)

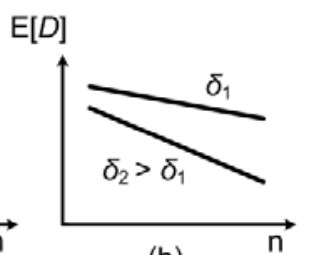

(b)

Fig. 1. Asymptotic representation of the expected message delay

where $\mathrm{N}_{2}$ denote the number of copies of the content at the time it reaches its destination. The distribution of the number of copies $P\left\{N_{2}=i\right\}$ is given by

$$
P\left\{N_{2}=i\right\}=\frac{1-(1-\delta)^{n-i}}{\delta(n-i)} \prod_{j=1}^{i-1}\left(1-\frac{1-(1-\delta)^{n-j}}{\delta(n-j)}\right) .
$$

The proofs of the proposition can be found in [14] and is derived from [6] adding parameter $\delta$.

The asymptotic representation of the expected message delay suggests that the role of interest is significant in the case of high node density while the role of contact rates becomes more important in the case of low density of nodes (Fig. 1). Consequently, improving the interest is rewarding if the number of mobile nodes is large, while improving contact rates provides better performance if the number of nodes is small. Using the results above, we can decide if the performance is acceptable using only contact opportunities between mobile users or if the addition of data kiosks becomes mandatory.

\subsection{Number of Data Kiosks}

We consider the situation where a hybrid solution is necessary to satisfy a given performance objective for the application. The performance metric is defined as the spreading time. An upper bound and a lower bound for the number of data kiosks are obtained under the following assumptions:

- In the case of the hybrid solution, we assume that mobile nodes do not change their interest in the lifetime of their journey (of course, they can add new interest),

- the number of data kiosks is greater than $\lambda / \mu$,

- subscribers do not hold a copy of the content at the beginning of their journey,

- the number of infected nodes is at least equal to two by the spreading time (one usually wants to have a lot of subscribers infected by the spreading time). 
The following notations are introduced:

- $m$ is the total number of subscribers at time 0 .

$-d$ is a performance objective for the expected spreading time.

$-b$ is the number of infected nodes by the spreading time.

Proposition 2. If the number of data kiosks is larger than $\lambda / \mu$, then an upper bound and a lower bound of the number of data kiosks to ensure that b subscribers will receive the content by the expected spreading time $d$ are given by $\lfloor L\rfloor<k<$ $\lceil U\rceil$,

$$
\begin{gathered}
U=\frac{\lambda}{2 \mu}+\frac{H+\sqrt{H^{2}+4 \lambda d\left(b_{\gamma}+1\right)(H+1)}}{2 \mu d} \\
L=\frac{-\lambda\left(b_{\gamma}+2 m\right)}{4 \mu}+\frac{2 \varsigma+\sqrt{\left[\lambda d\left(b_{\gamma}-2 m\right)+2 \varsigma\right]^{2}+8 \lambda d\left(2 \tilde{b}_{\gamma}+1\right)}}{4 \mu d}
\end{gathered}
$$

where $H=e^{-(0.5+m) \lambda d-\varsigma}-1, \varsigma=\sum_{i=0}^{b-1} \frac{1}{m-i}, b_{\gamma}=b+e^{-\gamma}-1.5, \tilde{b}_{\gamma}=b-e^{-\gamma}$, $\gamma$ is Euler-Mascheroni constant $\gamma \approx 0.57721$, e is Euler's number $e \approx 2.71828$. The proof is provided in section 4 .

In the formulae, if $b=m$, the computed number of data kiosks ensures that a subscriber will receive the content before $d$ on average. The following corollary is derived directly from Proposition 2.

Corollary 1. Suppose that the contact rate between a data kiosk and a mobile node in two distinct environments $A$ and $B$ is $\mu_{A}$ and $\mu_{B}$ respectively. Let $U_{A}$ and $L_{A}$ (resp. $U_{B}$ and $L_{B}$ ) be an upper bound and a lower bound of the number of data kiosks in the environment $A$ (resp. B). If we keep the same requirement for the expected spreading time, then a relationship between the bounds of the number of data kiosks and the contact rates in these environments is as follows:

$$
\frac{U_{B}}{U_{A}}=\frac{L_{B}}{L_{A}}=\frac{\mu_{A}}{\mu_{B}} .
$$

Corollary 1 shows that the bounds of the number of data kiosks scale linearly with the contact rates between a mobile node and a data kiosk.

\section{Analysis of the Number of Data Kiosks}

In this section, we focus on the analytical model to compute the number of data kiosks to meet a target for the expected spreading time.

We represent the number of copies of a given content in the network as an absorbing finite state Markov chain comprising $b+1$ states labeled by $\{0,1, \ldots, b\}$. The chain is in state $i$ when there are $i$ infected nodes in the network. If a current 
state $i$ is not the absorbing state $b$, the chain will make a transition into its next state $i+1$ after a time period. The spreading time that we are going to compute is the time until absorption of the chain.

We first compute the expected spreading time. A healthy node is infected the content when it meets either one of $k$ data kiosks or one of $i$ infected nodes. Then, a healthy node is infected with the content at an exponential rate $\lambda i+\mu k$.

The chain leaves state $i$ when one of $m-i$ healthy nodes is infected with the content. The event that a healthy node is infected occurs at an exponential rate $\lambda i+\mu k$, then the event that the chain will make a transition into its next state occurs at an exponential rate $(\lambda i+\mu k)(m-i)$. Let $T_{i}, 0 \leq i \leq b-1$, be the time interval in which the chain stays in state $i$. Since $T_{i}$ has an exponential distribution with parameter $(k \mu+i \lambda)(m-i)$, we have $E\left[T_{i}\right]=\frac{1}{(k \mu+i \lambda)(m-i)}$.

Let $T$ denote the spreading time, i.e. the time until there are $b$ infected nodes in the network. Then, $T=\sum_{i=0}^{b-1} T_{i}$ and $E[T]=E\left[\sum_{i=0}^{b-1} T_{i}\right]=\sum_{i=0}^{b-1} E\left[T_{i}\right]$.

We are ready to provide an expression for the expected spreading time $d$ :

$$
d=\sum_{i=0}^{b-1} \frac{1}{(k \mu+i \lambda)(m-i)} .
$$

We now have to compute the number of data kiosks to meet an objective for the expected spreading time $d$. Unfortunately, it is not possible to find a closedform expression for $m$ in the general case and we will therefore provide bounds. Lemma 1 to 4 will give the foundation to derive these bounds. Due to space limits, we only present the proof for Lemma 1. Details of the proofs for Lemma 2 to 4 can be found in [15].

Lemma 1. An expression of the expected spreading time represented under digamma function is

$$
d=\frac{\psi(u+b)-\psi(u)+\varsigma}{\lambda u+\lambda m}
$$

where $\varsigma=\sum_{i=0}^{b-1} \frac{1}{m-i}, u=\frac{\mu}{\lambda} k, \psi(x)$ is a digamma function of $x$.

Proof. Using an equation 6.3.16 of the digamma function in [1], we have $\psi(x+1)$ $=-\gamma+\sum_{i=1}^{\infty}\left(\frac{1}{i}-\frac{1}{x+i}\right)(x \neq-1,-2, \ldots)$ with $\gamma$ is Euler-Mascheroni constant $\gamma \approx 0.57721$. Since $k \geq \lambda / \mu$, we get $u=\mu k / \lambda \geq 1$. Applying the equation of the digamma function, we find $\psi(u+b)=-\gamma+\sum_{i=1}^{\infty}\left(\frac{1}{i}-\frac{1}{u+b-1+i}\right)$ and $\psi(u)=$ $-\gamma+\sum_{i=1}^{\infty}\left(\frac{1}{i}-\frac{1}{u-1+i}\right)$. Subtracting the later equation from the former, we get

$$
\psi(u+b)-\psi(u)=\sum_{i=1}^{b} \frac{1}{(u-1)+i}=\sum_{i=0}^{b-1} \frac{1}{u+i} .
$$


From (3), we have $d=\frac{1}{\lambda u+\lambda m}\left(\sum_{i=0}^{b-1} \frac{1}{u+i}+\varsigma\right)$ where $\varsigma=\sum_{i=0}^{b-1} \frac{1}{m-i}, u=\frac{\mu}{\lambda} k$. Substituting (5) into the equation, we get (4) which proves Lemma 1.

Lemma 2. With $u \geq 1$ and $b \geq 2$, inequalities of $\psi(u+b)-\psi(u)$ are

$$
\ln \left(1+\frac{\tilde{b}_{\gamma}+0.5}{u+e^{-\gamma}-1}\right)<\psi(u+b)-\psi(u)<\ln \left(1+\frac{b_{\gamma}+1}{u-0.5}\right)
$$

where $b_{\gamma}=b+e^{-\gamma}-1.5, \tilde{b}_{\gamma}=b-e^{-\gamma}$.

Lemma 3. One solution of the inequality $\lambda d u+\lambda d m-\varsigma<\ln \left(1+\frac{b_{\gamma}+1}{u-0.5}\right)$ is $\left\{u \in \mathbf{R} \mid u \geq 1, u<u_{2}\right\}$ where

$$
u_{2}=0.5+\frac{H+\sqrt{H^{2}+4 \lambda d\left(b_{\gamma}-0.5\right)(H+1)}}{2 \lambda d}, H=e^{-(0.5+m) \lambda d-\varsigma}-1 .
$$

Lemma 4. One solution of the inequality $\lambda d u+\lambda d m-\varsigma>\ln \left(1+\frac{\tilde{b}_{\gamma}+0.5}{u+e^{-\gamma}-1}\right)$ is $\left\{u \in \mathbf{R} \mid u \geq 1, u>u_{4}\right\}$ where

$$
u_{4}=\frac{-\left(b_{\gamma}+2 m\right)}{4}+\frac{2 \varsigma+\sqrt{\left[\lambda d\left(b_{\gamma}-2 m\right)+2 \varsigma\right]^{2}+8 \lambda d\left(2 \tilde{b}_{\gamma}+1\right)}}{4 \lambda d} .
$$

We are almost ready to derive bounds of the number of data kiosks. Using Lemma 1, we find $\lambda d u+\lambda d m-\varsigma=\psi(u+b)-\psi(u)$.

Because the number of data kiosks is larger than $\lambda / \mu$, it follows that $u \geq 1$. Suppose that the number of infected nodes is greater than or equal to two by the spreading time, $b \geq 2$. Hence, using Lemma 2 , we get $\ln \left(1+\frac{\tilde{b}_{\gamma}+0.5}{u+e^{-\gamma}-1}\right)<$ $\lambda d u+\lambda d m-\varsigma<\ln \left(1+\frac{b_{\gamma}+1}{u-0.5}\right)$. From Lemma 3 and Lemma 4, we obtain a solution for the inequalities. Substituting $u=\frac{k \mu}{\lambda}$ into the solution, we find an upper bound and a lower bound of the number of data kiosks $\lfloor L\rfloor<k<\lceil U\rceil$, where $U$ is represented by (11) and $L$ is described by (2), which demonstrates Proposition 2.

\section{Validation}

In this section, we validate the theoretical results through simulation under three mobility models, and discuss the application of the results. For a given context and a delay objective, we can state if the delay is acceptable to deploy the service. If negative, we provide the number of data kiosks that should be added in a hybrid DTN environment to meet the requirement. 

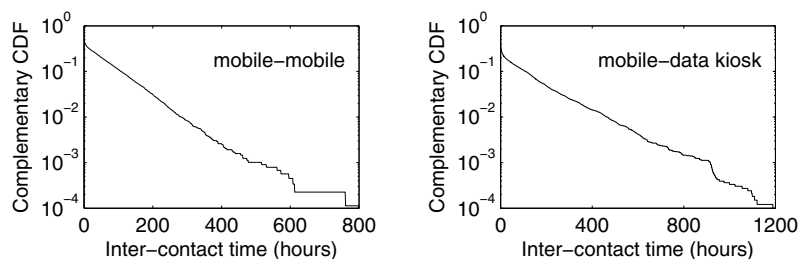

Fig. 2. CCDF of inter-contact time for the random walker mobility model

\subsection{Simulation Settings}

We simulated the user-preferred content distribution scheme under the random waypoint, random direction and random walker mobility models in the Opportunistic Network Environment [10. Mobile nodes move according to the mobility models under consideration and data kiosks are uniformly located in a square of size $4 \mathrm{~km} \times 4 \mathrm{~km}$. Radio ranges of nodes are 50 meters.

In the random waypoint mobility model, a node travels to a destination chosen uniformly in an area with a constant speed. The speed of a mobile node (in $\mathrm{km} / \mathrm{h}$ ) is chosen uniformly in $\left[v_{\min }, v_{\max }\right]=[4,10]$. Upon arrival, the node continues its trip by choosing a new destination and a new speed, independently of all previous destinations and speeds.

In the random direction mobility model, a node selects an initial direction, speed and a finite travel time. The node then travels in the direction at the speed for the duration. Once this time expires, it chooses a new direction, speed and travel time which are independent of all its past directions, speeds and travel times. When a node reaches a boundary it is reflected. In our setting, the speed of a mobile node $\left(\mathrm{in} \mathrm{km} / \mathrm{h}\right.$ ) is chosen uniformly in $\left[v_{\min }, v_{\max }\right]=[4,10]$, a direction is uniformly distributed in $[0,2 \pi)$, and travel time is exponentially distributed with mean $1 / 4$ hour.

In the two-dimensional random walker mobility model, a node moves on a square grid at a constant speed. At crossroads, a node uniformly chooses to go to a next crossroad in the front, in the back, on the left, or on the right. When a node has reached a crossroad at the borders, it will go to the opposite crossroad if the newly chosen crossroad is out of the borders. We set the distance among adjacent crossroads to $80 \mathrm{~m}$ and a constant speed of $4.8 \mathrm{~km} / \mathrm{h}$.

For the random waypoint and random direction mobility model, formulae for $\lambda$ and $\mu$ were introduced 67 . Using the parameter settings of our simulation, we computed the values for $\lambda$ and $\mu$ and found that the contact rates are $\lambda \approx$ 0.0741 and $\mu \approx 0.0409$ for the random waypoint mobility model, $\lambda \approx 0.0577$ and $\mu \approx 0.0437$ for the random direction mobility model.

A mathematical formula for the exponential tail under the random walker mobility model is, to the best of our knowledge, not known. Therefore we obtained its value through simulation. Fig. 2 plots the complementary cumulative distribution function $(\mathrm{CCDF})$ of the inter-contact time on log-lin scale. Estimates of contact rates for this last mobility model are $\lambda \approx 0.0127$ and $\mu \approx 0.0070$. 


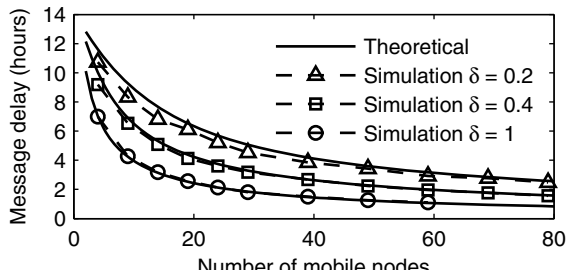

(a) Random waypoint: $r=50 \mathrm{~m}$

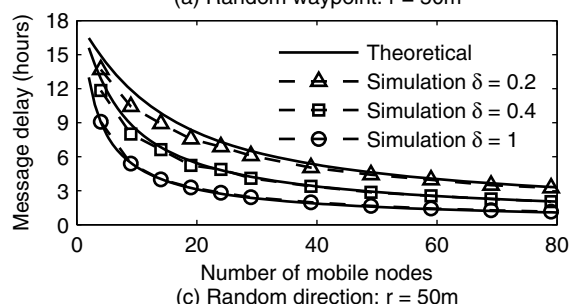

(c) Random direction: $r=50 \mathrm{~m}$

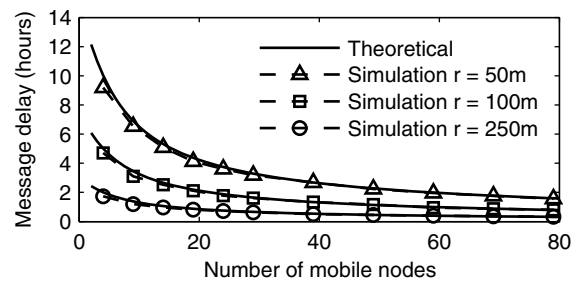

(b) Random waypoint: $\delta=0.4$

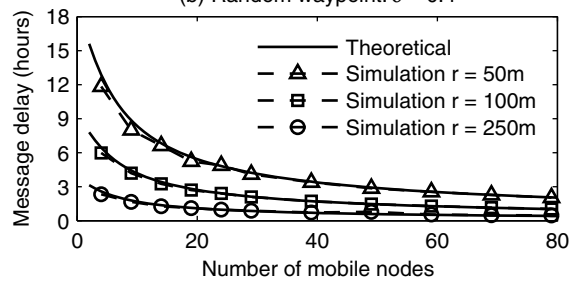

(d) Random direction: $\delta=0.4$

Fig. 3. Message delay vs. number of mobile nodes: Random waypoint, random direction mobility model

\subsection{Lower Delay to a Requirement}

First, we consider the expected message delay in the situation with zero data kiosk. For the three probability of interest $(\delta=1,0.4,0.2)$, and under the three mobility models, we varied the number of nodes between 5 and 80 , and computed the average message delay based on 1000 observations obtained from simulation for each configuration. Figs. 3,4 plot the expected message delay as a function of the number of nodes, comparing the theoretical analyses with the simulation results. They confirm the accuracy of the theoretical analysis for these mobility models. Although the inter-contact time is only exponential tail under the random walker mobility model as we see in Fig. 2, the theoretical analysis still can predict the message delay for the different probability of interest and the different contact rates. The error between simulation and theoretical results increases when the probability of interest is small.

Next, in the hybrid case, we run simulations for different number of subscribers $(k=40,60)$. The number of data kiosks was varied between 2 to 20 . For each setting, we ran 1000 observations and computed the expected spreading time by which the content is delivered to 90 percent of subscribers. Then, we used analytical expressions (1) and (2) to calculate an upper bound and a lower bound of the number of data kiosks for a value of the average spreading time that we found from simulation. For each spreading time, we compared the estimated values of the number of data kiosks provided thanks to the theoretical solution or by simulation. Fig. 5 depicts the results under the three mobility models.

We observe that analytical results can approximately predict the number of data kiosks, but the lower bounds deteriorate as the expected spreading time gets very small which is certainly not meaningful. Finally, we give an example for the application of our results with ideal parameters. We first consider the 

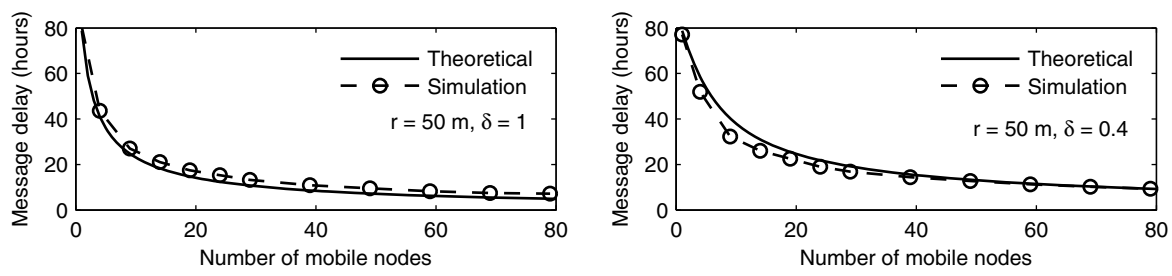

Fig. 4. Message delay vs. number of mobile nodes: Random walker mobility model

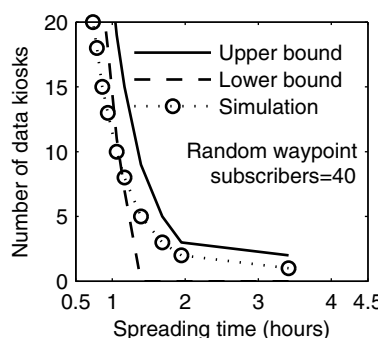

(a)

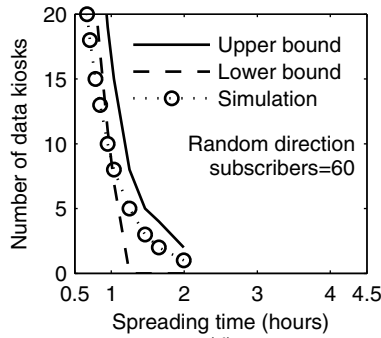

(d)

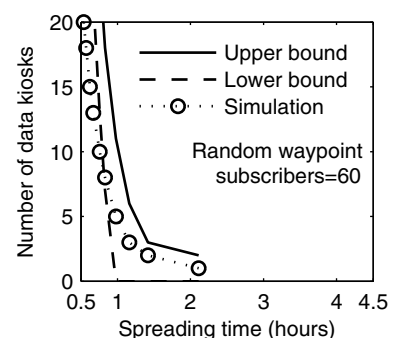

(b)

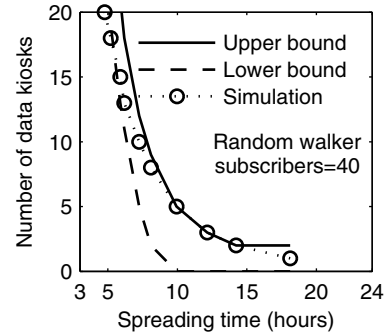

(e)

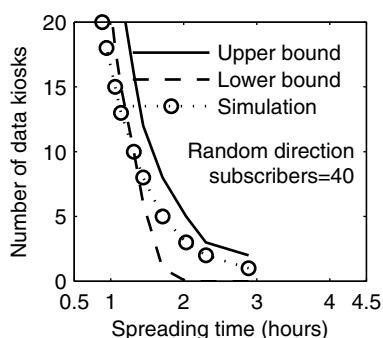

(c)

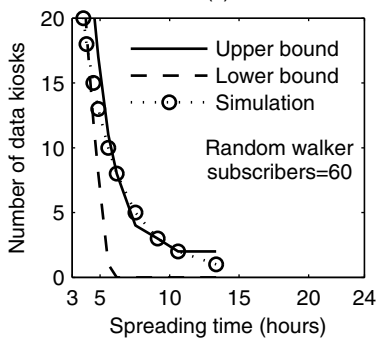

(f)

Fig. 5. Number of data kiosks vs. spreading time

scenario without data kiosk. The urban area is a square of size $4 \mathrm{~km} \times 4 \mathrm{~km}$. There are 40 mobile nodes moving under the random waypoint mobility model. The transmission range is 50 meters, the probability of interest is 1 , as every node is interested in the content. Under this scenario, the expected message delay is roughly 2 hours (as in Fig. [3(a)). If we wish to spread the content to 90 percent of the subscribers within 1 hour, it appears difficult to satisfy this requirement in a network without data kiosk. We now consider an hybrid environment. We keep these settings and find the number of data kiosks that we should add to meet the requirement. As we see in Fig. 5(a), with 10 data kiosks we can lessen the expected spreading time to 1 hour. We also see that the spreading time is improved effectively with some data kiosks. For example, with 5 data kiosks the expected spreading time can decrease to 2 hours in the random waypoint mobility model (as in Fig. 5(a)), and approximately to less than 8 hours in the random walker mobility model (see Fig. [5(e-f)). 


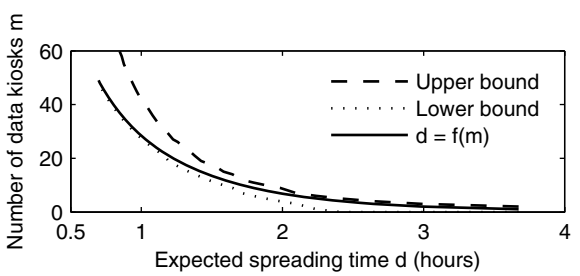

Fig. 6. Bounds of the number of data kiosks and the function of expected spreading time

The simulation results confirm that the analytical models can predict the expected message delay and the number of data kiosks to meet a delay requirement. The lower bound of the number of data kiosks gets close to the simulation results when the expected spreading time is small and the upper bound is accurate when the expected spreading time is large (Fig. 6).

\section{Conclusion}

In this paper, we investigated the practicality of dissemination of daily news over a DTN in an urban area. When the performance can not fulfill a requirement, a solution is to deploy a hybrid environment with data kiosks to overcome the limit of the performance of DTNs. We obtained both a closed-form and an approximation expression of the expected message delay. The results were used to analyze the influence of user's interest and contact rates on the performance of DTNs. If the interest of people does not vary over the spreading time, we compute the solution for the number of data kiosks that we need to deploy to satisfy an expected spreading time objective. The results show that the message delay in DTNs is in the range of several hours but can be significantly reduced thanks to the addition of data kiosks. One question that we wish to address in our future work is where data kiosks should be located to maximize the contact opportunity between data kiosks and mobile users, or to minimize the delay. The work can also be extended by taking into account interferences, packet sizes and transmission times.

Acknowledgments. This work was supported in part by the Agence nationale de la recherche - ANR - under the CROWD project:

http://anr-crowd.lip6.fr/.

\section{References}

1. Abramowitz, M., Stegun, I.A.: Handbook of Mathematical Functions: with Formulas, Graphs, and Mathematical Tables. Dover Publications, New York (1965)

2. Al Hanbali, A., Kherani, A.A., Groenevelt, R., Nain, P., Altman, E.: Impact of mobility on the performance of relaying in ad hoc networks - extended version. Comput. Netw. 51(14), 4112-4130 (2007) 
3. Al Hanbali, A., Nain, P., Altman, E.: Performance of ad hoc networks with two-hop relay routing and limited packet lifetime (extended version). Perform. Eval. 65(6-7), 463-483 (2008)

4. Banerjee, N., Corner, M.D., Towsley, D., Levine, B.N.: Relays, base stations, and meshes: enhancing mobile networks with infrastructure. In: MobiCom 2008: Proceedings of the 14th ACM International Conference on Mobile Computing and Networking, pp. 81-91. ACM, New York (2008)

5. Garyfalos, A., Almeroth, K.: Coupons: A multilevel incentive scheme for information dissemination in mobile networks. IEEE Transactions on Mobile Computing 7(6), 792-804 (2008)

6. Groenevelt, R., Nain, P., Koole, G.: The message delay in mobile ad hoc networks. Perform. Eval. 62(1-4), 210-228 (2005)

7. Ibrahim, M., Al Hanbali, A., Nain, P.: Delay and resource analysis in manets in presence of throwboxes. Perform. Eval. 64(9-12), 933-947 (2007)

8. Ioannidis, S., Chaintreau, A., Massoulié, L.: Optimal and scalable distribution of content updates over a mobile social network. In: INFOCOM 2009, pp. 1422-1430. IEEE, Los Alamitos (2009)

9. Karagiannis, T., Le Boudec, J.Y., Vojnović, M.: Power law and exponential decay of inter contact times between mobile devices. In: MobiCom 2007: Proceedings of the 13th Annual ACM International Conference on Mobile Computing and Networking, pp. 183-194. ACM, New York (2007)

10. Keränen, A., Ott, J., Kärkkäinen, T.: The ONE simulator for DTN protocol evaluation. In: Simutools 2009: Proceedings of the 2nd International Conference on Simulation Tools and Techniques. ICST (Institute for Computer Sciences, SocialInformatics and Telecommunications Engineering), pp. 1-10. ICST, Brussels (2009)

11. Lenders, V., May, M., Karlsson, G., Wacha, C.: Wireless ad hoc podcasting. SIGMOBILE Mob. Comput. Commun. Rev. 12, 65-67 (2008)

12. Liu, B., Thiran, P., Towsley, D.: Capacity of a wireless ad hoc network with infrastructure. In: MobiHoc 2007: Proceedings of the 8th ACM International Symposium on Mobile Ad Hoc Networking and Computing, pp. 239-246. ACM, New York (2007)

13. McNamara, L., Mascolo, C., Capra, L.: Media sharing based on colocation prediction in urban transport. In: MobiCom 2008: Proceedings of the 14th ACM International Conference on Mobile Computing and Networking, pp. 58-69. ACM, New York (2008)

14. Pham, T.M., Fdida, S.: Delay estimation of a user-preferred content distribution scheme in disruption tolerant networks. In: AINTEC 2009: Asian Internet Engineering Conference, pp. 3-10. ACM, New York (2009)

15. Pham, T.M., Fdida, S.: DTN support for news dissemination in an urban area. Technical report hal-00565307, UPMC (2011), http://hal.upmc.fr/hal-00565307/en/

16. Polat, B.K., Sachdeva, P., Ammar, M.H., Zegura, E.W.: Message ferries as generalized dominating sets in intermittently connected mobile networks. In: MobiOpp 2010: Proceedings of the Second International Workshop on Mobile Opportunistic Networking, pp. 22-31. ACM, New York (2010)

17. Robinson, J., Singh, M., Swaminathan, R., Knightly, E.: Deploying mesh nodes under non-uniform propagation. In: Proceedings of the 29th Conference on Information Communications, INFOCOM 2010, pp. 2142-2150. IEEE Press, Piscataway (2010) 
18. Sharma, G., Mazumdar, R., Shroff, N.B.: Delay and capacity trade-offs in mobile ad hoc networks: a global perspective. IEEE/ACM Trans. Netw. 15(5), 981-992 (2007)

19. Spyropoulos, T., Psounis, K., Raghavendra, C.S.: Spray and wait: an efficient routing scheme for intermittently connected mobile networks. In: WDTN 2005: Proceedings of the 2005 ACM SIGCOMM Workshop on Delay-tolerant Networking, pp. 252-259. ACM, New York (2005)

20. Zemlianov, A., Gustavo de, V.: Capacity of ad hoc wireless networks with infrastructure support. IEEE Journal on Selected Areas in Communications 23(3), 657-667 (2005)

21. Zhang, X., Neglia, G., Kurose, J., Towsley, D.: Performance modeling of epidemic routing. Comput. Netw. 51(10), 2867-2891 (2007) 